Article

\title{
Acute Respiratory Distress Syndrome and Time to Weaning Off the Invasive Mechanical Ventilator among Patients with COVID-19 Pneumonia
}

\author{
Jose Bordon 1,2,3,*, Ozan Akca 3,4,5,6 , Stephen Furmanek ${ }^{3,4,6}$, Rodrigo Silva Cavallazzi ${ }^{3,7}$, Sally Suliman ${ }^{7}$, \\ Amr Aboelnasr ${ }^{3,4,6}$, Bettina Sinanova ${ }^{1}$ and Julio A. Ramirez ${ }^{3,4,6}$ \\ 1 Washington Health Institute, Washington, DC 20017, USA; bettina_sinanova@yahoo.com \\ 2 Department of Medicine, George Washington University Medical Center, Washington, DC 20037, USA \\ 3 Center of Excellence for Research in Infectious Diseases (CERID), University of Louisville, \\ Louisville, KY 40292, USA; ozan.akca@louisville.edu (O.A.); stephen.furmanek@louisville.edu (S.F.); \\ rodrigo.cavallazzi@louisville.edu (R.S.C.); amr.aboelnasr@louisville.edu (A.A.); \\ j.ramirez@louisville.edu (J.A.R.) \\ 4 Department of Anesthesiology and Perioperative Medicine, University of Louisville, \\ Louisville, KY 40292, USA \\ 5 Comprehensive Stroke Clinical Research Program (CSCRP), University of Louisville, \\ Louisville, KY 40292, USA \\ 6 Division of Infectious Diseases, University of Louisville, Louisville, KY 40292, USA \\ 7 Division of Critical Care Medicine, University of Louisville, Louisville, KY 40292, USA; \\ sally.suliman@louisville.edu \\ check for \\ updates \\ * Correspondence: jbordon@dc-whi.org; Tel.: +1-240-426-8890
}

Citation: Bordon, J.; Akca, O.; Furmanek, S.; Cavallazzi, R.S.; Suliman, S.; Aboelnasr, A.; Sinanova, B.; Ramirez, J.A. Acute Respiratory Distress Syndrome and Time to Weaning Off the Invasive Mechanical Ventilator among Patients with COVID-19 Pneumonia. J. Clin. Med. 2021, 10, 2935. https://doi.org/ $10.3390 /$ jcm10132935

Academic Editor: Davide Chiumello

Received: 13 June 2021

Accepted: 26 June 2021

Published: 30 June 2021

Publisher's Note: MDPI stays neutral with regard to jurisdictional claims in published maps and institutional affiliations.

Copyright: (c) 2021 by the authors. Licensee MDPI, Basel, Switzerland. This article is an open access article distributed under the terms and conditions of the Creative Commons Attribution (CC BY) license (https:// creativecommons.org/licenses/by/ $4.0 /)$.

\begin{abstract}
Acute respiratory distress syndrome (ARDS) due to coronavirus disease 2019 (COVID-19) pneumonia is the main cause of the pandemic's death toll. The assessment of ARDS and time on invasive mechanical ventilation (IMV) could enhance the characterization of outcomes and management of this condition. This is a city-wide retrospective study of hospitalized patients with COVID-19 pneumonia from 5 March 2020 to 30 June 2020. Patients with critical illness were compared with those with non-critical illness. We examined the severity of ARDS and other factors associated with (i) weaning patients off IMV and (ii) mortality in a city-wide study in Louisville, KY. Of 522 patients with COVID-19 pneumonia, 219 (41.9\%) were critically ill. Among critically ill patients, the median age was 60 years; $53 \%$ were male, $55 \%$ were White and $32 \%$ were African American. Of all critically ill patients, $52 \%$ had ARDS, and $38 \%$ of these had severe ARDS. Of the $25 \%$ of patients who were weaned off IMV, those with severe ARDS were weaned within eleven days versus five days for those without severe ARDS, $p=0.023$. The overall mortality for critically ill patients was $22 \%$ versus $1 \%$ for those not critically ill. Furthermore, the 14 -day mortality was $31 \%$ for patients with severe ARDS and $12 \%$ for patients without severe ARDS, $p=0.019$. Patients with severe ARDS versus non-severe ARDS needed twice as long to wean off IMV (eleven versus five days) and had double the 14-day mortality of patients without severe ARDS.
\end{abstract}

Keywords: COVID-19; SARS-CoV-2; CAP; pneumonia; ARDS; IMV

\section{Introduction}

As of 22 June 2021, there have been 3,876,081 losses of human life reported worldwide due to the COVID-19 pandemic [1]. COVID-19 pneumonia resulting in critical illness is the main cause of the high death toll of this pandemic. Up to $39.7 \%$ of patients with COVID-19 have been reported to develop critical illness-primarily associated with acute respiratory distress syndrome (ARDS) —in a predominantly African American cohort [2]. Furthermore, the mortality rate of critically ill patients with COVID-19 has been reported at up to $98 \%$ [3]. The characteristics and major outcomes of critically ill patients due to COVID-19 pneumonia on a US city-wide scale have not yet been reported. ARDS is a 
major outcome of COVID-19 patients that requires a remarkably long invasive mechanical ventilation (IMV). The time to wean patients off IMV is a turning point in prognosis, at which point aggressive clinical management can be de-escalated.

Indeed, it is critically important to determine the associated factors and the average time to wean COVID-19 patients off of IMV. An optimal characterization of critically ill patients with COVID-19 from a population representative of the US as a whole could provide balanced data for the development of guidelines regarding the appropriate assessment and management of COVID-19. The city of Louisville, KY, has been recognized as a population with sociodemographic, economic, and health-related characteristics that are representative of the US [4]. The 2019 population of the city of Louisville was 766,557, of whom $16.7 \%$ were persons 65 years and older, $51.7 \%$ were women, $71.8 \%$ were Caucasians, and $22.4 \%$ were African Americans; moreover, median house income was $\$ 54,357$ and $15.4 \%$ of the population was living in poverty [5].

We measured the outcomes of patients who were critically ill due to COVID-19 pneumonia; we examined the severity of ARDS and factors associated with (i) weaning patients off IMV and (ii) mortality in a city-wide study in Louisville, KY, USA.

\section{Methods}

\subsection{Study Design, Subjects, and Setting}

This study was a secondary data analysis of the Louisville Coronavirus Surveillance Program, KY, USA, a city-wide retrospective observational study of consecutive unduplicated adult patients with COVID-19 pneumonia who required admission to any of the city's 8 hospitals between 5 March 2020 - when the first hospitalized patient with COVID-19 was identified in Louisville-and 1 July 2020 [6]. Our study group comprised patients with COVID-19 pneumonia who were critically ill. The control group consisted of patients with COVID-19 pneumonia who were not critically ill and were admitted to any of the eight hospitals in the city of Louisville, KY, USA. Patient follow-up ceased at hospital discharge. We examined the severity of ARDS and other factors associated with (i) weaning patients off IMV, and (ii) mortality in a city-wide study in Louisville, KY. Supplementary File S1.

\subsection{Severity of Illness Definitions}

The severity of illness was defined during the first $24 \mathrm{~h}$ of hospitalization in accordance with National Institutes of Health (NIH) guidelines for the management of COVID-19 [7]. Only patients with Stages 3 to 9 COVID-19 were included in this study, and Stages 6 to 9 were considered critical illness. In the cases of patients with critical illness, the analysis used data recorded from the point at which they fulfilled the criteria of having critical illness, either at the time of hospitalization or thereafter.

\subsection{Mortality Prediction}

COVID-19 pneumonia at the time of hospitalization and mortality prediction were evaluated using two well-established pneumonia scores, the pneumonia severity index (PSI) score [8] and the CURB-65 score [9].

\subsection{Exclusion Criteria}

Patients without COVID-19 and patients who did not require hospital admission were excluded from this study. Patients who had do-not-resuscitate (DNR) or do-not-intubate (DNI) orders upon admission and individuals younger than 18 years were also excluded.

\subsection{Predictor Variables}

Predictor variables included clinical characteristics, laboratory findings, and imaging results at the time of hospitalization. In cases of critical SARS-CoV-2 CAP, these variables were collected within $24 \mathrm{~h}$ of the time at which the patients fulfilled the definition of critical COVID-19, either at hospital admission or during hospitalization. 


\subsection{Outcome Variables}

i. Time to wean off IMV was truncated at 21 days. Weaning success was eliminated if the patient died soon thereafter. Patients who remained on IMV, or were weaned off but expired while in the hospital, were considered instances of failure to wean off IMV. In the event that a patient weaned off IMV died in the hospital, the time to wean off invasive mechanical ventilation was censored and set to 21 days. Patients who underwent tracheostomy were not considered weaned until after decannulation.

ii. Time to mortality was truncated at 30 days. Patients who were discharged alive before 30 days of hospitalization were not followed after discharge and were given a rightcensored time to mortality consisting of the duration at their date of hospital discharge.

\subsection{Statistical Analysis}

Patient characteristics were reported as frequencies and percentages or as medians and interquartile ranges, for categorical and continuous data, respectively. Differences in baseline patient characteristics were assessed by chi-squared tests of independence or Mann-Whitney $U$ tests for categorical and continuous data, respectively.

Inflammatory laboratory values of lactate, ferritin, interleukin-6 (IL-6), D-dimer, and creactive protein (CRP) were dichotomized into high/low values based on receiver operating curve (ROC) values that maximized sensitivity and specificity to predict in-hospital mortality. Time-to-event data were represented by Kaplan-Meier curves split by severity of ARDS. Log-rank tests were performed for unadjusted comparisons. To determine protective and risk factors for time to event data, multivariable, stratified Cox proportional hazards regressions with cluster-robust standard errors were performed. Variables were selected through forward and backward stepwise selection, based on minimizing the Akaike information criterion (AIC). The variables used in the selection process were (i) patient demographics of age, sex, race, and ethnicity; (ii) history of the following variables: neoplastic disease within the past year, renal disease, heart failure, chronic obstructive pulmonary disease, smoking history, diabetes, obesity, hypertension, hyperlipidemia; (iii) standardized values of temperature, respiratory rate, mean arterial pressure, heart rate, blood urea nitrogen (BUN), serum glucose, serum sodium, and hematocrit; (iv) dichotomized laboratory values of IL-6, CRP, d-dimer, ferritin, and lactate; and (v) exam findings of altered mental status and computed tomography $(\mathrm{CT})$ or chest $\mathrm{x}$-ray findings of pleural effusion and severe ARDS. Any $p$-value less than 0.05 was considered statistically significant. All analysis was performed in $\mathrm{R}$ version 3.5.1. (Vienna, Austria.)

\section{Results}

\subsection{Study Population and Main Characteristics}

Of the 712 patients hospitalized due to COVID-19 from 5 March 2020 to 30 June 2020 in the city of Louisville, KY, our study included a total of 522 patients with COVID19 pneumonia (Figure 1). Due to DNR and DNI advanced directives, 67 critically ill patients and 43 non-critically ill patients were excluded. Our study included a final total of 219 critically ill and 303 non-critically ill COVID-19 pneumonia patients. The main characteristics of these patients are shown in Table 1. 


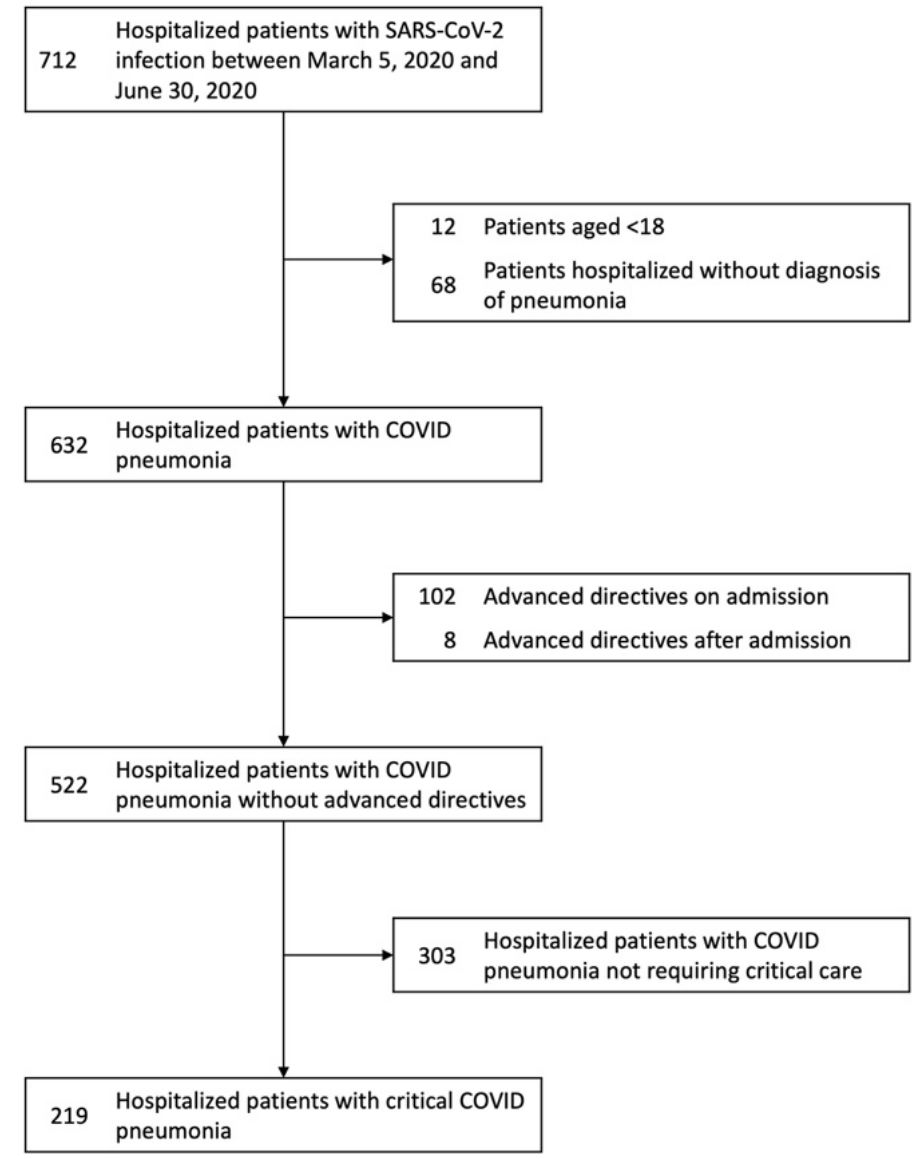

Figure 1. Flowchart of patients with COVID-19 hospitalized in the city of Louisville, KY, from 5 March 2020 to 30 June 2020. The 219 patients with critical COVID-19 were selected upon elimination of 67 patients with do-not-resuscitate and do-not-intubate advanced directive orders. Similarly, the 303 patients with non-critical COVID-19 pneumonia resulted in the elimination of 43 patients. Abbreviations: COVID-19, coronavirus disease 2019; DNI, do-not-intubate; DNR, do-not-resuscitate; SARS-CoV-2, severe acute respiratory syndrome coronavirus-2.

Table 1. Characteristics of patients with COVID-19 pneumonia at the time of assignment to the groups of critical and non-critical illness.

\begin{tabular}{|c|c|c|c|}
\hline & $\begin{array}{l}\text { Critical Illness } \\
\quad(n=219)\end{array}$ & $\begin{array}{l}\text { Non-Critical Illness } \\
\qquad(n=303)\end{array}$ & $p$ Value \\
\hline \multicolumn{4}{|l|}{ Demographics } \\
\hline Age (median (IQR)) & $60(50,70)$ & $58(44,71)$ & 0.234 \\
\hline Male sex $(\%)$ & $116(53)$ & $129(43)$ & 0.024 \\
\hline Race (\%) & & & 0.170 \\
\hline White & $121(55)$ & $144(48)$ & \\
\hline Black & $69(32)$ & $105(35)$ & \\
\hline Other & $29(13)$ & $54(18)$ & \\
\hline Hispanic ethnicity (\%) & $21(10)$ & $50(17)$ & 0.032 \\
\hline Neoplastic disease (\%) & $14(6)$ & $14(5)$ & 0.490 \\
\hline Renal disease $(\%)$ & $34(16)$ & $49(16)$ & 0.938 \\
\hline Liver disease (\%) & $4(2)$ & $13(4)$ & 0.188 \\
\hline Heart failure (\%) & $28(13)$ & $29(10)$ & 0.308 \\
\hline COPD $(\%)$ & $33(15)$ & $33(11)$ & 0.199 \\
\hline Smoking history, current or former $(\%)$ & $82(37)$ & $100(33)$ & 0.338 \\
\hline Diabetes $(\%)$ & $79(36)$ & $84(28)$ & 0.053 \\
\hline Obese (\%) & $121(55)$ & $154(51)$ & 0.362 \\
\hline
\end{tabular}


Table 1. Cont.

\begin{tabular}{|c|c|c|c|}
\hline & $\begin{array}{l}\text { Critical Illness } \\
\quad(n=219)\end{array}$ & $\begin{array}{l}\text { Non-Critical Illness } \\
\qquad(n=303)\end{array}$ & $p$ Value \\
\hline Hypertension $(\%)$ & $116(53)$ & $155(51)$ & 0.749 \\
\hline Coronary artery disease (\%) & $24(11)$ & $40(13)$ & 0.525 \\
\hline Hyperlipidemia (\%) & $72(33)$ & $97(32)$ & 0.910 \\
\hline \multicolumn{4}{|c|}{ Exam and lab values at time of admission or critical illness, median (IQR) } \\
\hline Temperature (degrees celsius) & $37.6(36.9,38.8)$ & $37.8(37.0,38.5)$ & 0.880 \\
\hline Respiratory rate (breaths/min) & $27.0(21.0,34.0)$ & $20.0(18.0,24.0)$ & $<0.001$ \\
\hline Heart rate (beats $/ \mathrm{min}$ ) & $101.0(87.5,112.5)$ & $97.0(80.0,110.0)$ & 0.050 \\
\hline Systolic blood pressure (mmHg) & $110.0(97.0,132.0)$ & $124.0(109.0,141.0)$ & $<0.001$ \\
\hline Diastolic blood pressure (mmHg) & $61.0(48.0,75.0)$ & $69.0(61.0,81.5)$ & $<0.001$ \\
\hline Mean arterial pressure (mmHg) & $77.7(64.7,92.3)$ & $88.7(78.3,99.0)$ & $<0.001$ \\
\hline $\mathrm{BUN}(\mathrm{mg} / \mathrm{dL})$ & $17.0(12.0,29.0)$ & $15.0(10.0,23.0)$ & 0.021 \\
\hline Glucose (mg/dL) & $132.0(112.0,183.0)$ & $117.0(102.0,143.0)$ & $<0.001$ \\
\hline Hematocrit $(\%)$ & $37.4(33.0,40.7)$ & $39.0(35.9,42.6)$ & $<0.001$ \\
\hline Sodium $(\mathrm{mEq} / \mathrm{L})$ & $136.0(133.0,138.0)$ & $136.0(134.0,138.0)$ & 0.544 \\
\hline White blood cell count $(\times 1000$ per $\mathrm{uL})$ & $7.8(5.8,11.0)$ & $5.6(4.3,7.3)$ & $<0.001$ \\
\hline Neutrophil count $(\times 1000$ per $\mathrm{uL})$ & $5.8(4.5,8.3)$ & $3.7(2.7,5.6)$ & $<0.001$ \\
\hline Lymphocyte count $(\times 1000$ per uL) & $0.9(0.7,1.1)$ & $1.1(0.8,1.4)$ & $<0.001$ \\
\hline Neutrophil/lymphocyte (ratio) & $8.0(5.2,10.1)$ & $3.4(2.2,5.9)$ & $<0.001$ \\
\hline Interleukin-6 (pg/mL) & $92.2(51.3,218.1)$ & $33.8(16.6,58.2)$ & $<0.001$ \\
\hline C-reactive protein $(\mathrm{CRP})(\mathrm{mg} / \mathrm{L})$ & $162.4(82.2,216.4)$ & $53.0(24.5,120.0)$ & $<0.001$ \\
\hline D-dimer $(\mathrm{ng} / \mathrm{mL})$ & $813.0(318.2,2147.5)$ & $681.0(370.0,1080.0)$ & 0.115 \\
\hline Ferritin $(\mathrm{ng} / \mathrm{mL})$ & $554.5(289.8,994.8)$ & $292.0(115.5,637.0)$ & $<0.001$ \\
\hline Lactate (mmol/L) & $1.5(1.2,2.4)$ & $1.2(1.0,1.7)$ & $<0.001$ \\
\hline Creatinine (mg/dL) & $1.0(0.7,1.4)$ & $0.9(0.7,1.2)$ & 0.024 \\
\hline Albumin (g/dL) & $3.3(2.8,3.6)$ & $3.8(3.5,4.1)$ & $<0.001$ \\
\hline Bilirubin (mg/dL) & $0.7(0.5,1.0)$ & $0.6(0.4,0.9)$ & $<0.001$ \\
\hline $\mathrm{PaO}_{2} / \mathrm{FiO}_{2}$ (ratio) & $174.0(85.4,253.0)$ & $355.0(336.8,378.5)$ & $<0.001$ \\
\hline $\mathrm{SpO}_{2} / \mathrm{FiO}_{2}$ (ratio) & $220.0(95.8,332.1)$ & $442.9(384.5,457.1)$ & $<0.001$ \\
\hline \multicolumn{4}{|l|}{ Treatments and therapies, $n(\%)$} \\
\hline Hydroxychloroquine (\%) & $120(55)$ & $76(25)$ & $<0.001$ \\
\hline Azithromycin $(\%)$ & $158(72)$ & $181(60)$ & 0.001 \\
\hline Remdesivir (\%) & $21(10)$ & $7(2)$ & $<0.001$ \\
\hline Steroids $(\%)$ & $91(42)$ & $28(9)$ & $<0.001$ \\
\hline Low molecular weight heparins (\%) & $150(68)$ & $180(59)$ & 0.092 \\
\hline Heparin $(\%)$ & $69(32)$ & $17(6)$ & $<0.001$ \\
\hline Plasma (\%) & $36(16)$ & $7(2)$ & $<0.001$ \\
\hline
\end{tabular}

Patients with advanced directives were not included. Abbreviations: BUN, blood urea nitrogen; COPD, chronic obstructive pulmonary disease; COVID-19, coronavirus disease 2019; IQR, interquartile range; $\mathrm{PaO}_{2} / \mathrm{FiO}_{2}$, arterial partial pressure of oxygen/fraction of inspired oxygen; $\mathrm{SpO}_{2} / \mathrm{FiO}_{2}$, peripheral blood oxygen saturation/fraction of inspired oxygen.

On average, critically ill COVID-19 patients were two years older than controls. The population of critically ill male patients was $10 \%$ greater than non-critically ill male patients (53\% versus. $43 \% p=0.02)$. Among critically ill pneumonia patients, African Americans accounted for 32\% and Caucasians 55\%. Arterial hypertension was present in $58 \%$ of critically ill versus $54 \%$ of non-critically ill patients, and diabetes mellitus was observed in $40 \%$ versus $29 \%$, respectively (Table 1 ).

\subsection{Severity of Illness and Outcomes}

Overall, critically ill patients with COVID-19 had greater severity of illness than controls, with the most notable difference being the degree of hypoxemia (Table 1); these differences were determined to be statistically significant. The unadjusted outcomes are shown in Table 2. ARDS was present in $52 \%$ and shock in $27 \%$ of critically ill patients. Cardiovascular events were reported more than three times more often among critically ill patients than those not critically ill: $27 \%$ versus $8 \%$ respectively. 
Table 2. Clinical outcomes among all patients hospitalized with COVID-19 pneumonia.

\begin{tabular}{|c|c|c|c|}
\hline & $\begin{array}{l}\text { Critical Illness } \\
\quad(n=219)\end{array}$ & $\begin{array}{l}\text { Non-Critical Illness } \\
\qquad(n=303)\end{array}$ & $p$ Value \\
\hline \multicolumn{4}{|l|}{ Outcomes } \\
\hline Time to death or discharge from admission & $14.0(8.0,20.0)$ & $4.1(2.6,6.9)$ & $<0.001$ \\
\hline Time to ICU admission from admission & $1.0(0.0,2.5)$ & & \\
\hline Time to death or ICU discharge from ICU admission & $8.3(2.7,15.2)$ & & \\
\hline Time to IMV from admission & $1.7(0.3,3.7)$ & & \\
\hline Time to death, tracheostomy, or weaning from intubation & $8.8(4.0,14.2)$ & & \\
\hline Time to IMV from ICU admission & $0.1(0.0,1.4)$ & & \\
\hline Septic shock $(\%)$ & $60(27)$ & $0(0)$ & $<0.001$ \\
\hline DIC $(\%)$ & $2(1)$ & $0(0)$ & 0.343 \\
\hline Development of ARDS (\%) & $114(52)$ & $0(0)$ & $<0.001$ \\
\hline Cardiovascular or cardiac event (\%) & $59(27)$ & $24(8)$ & $<0.001$ \\
\hline In-hospital mortality (\%) & $43(20)$ & $4(1)$ & $<0.001$ \\
\hline Age & $66(57,73)$ & $83(75,90)$ & $<0.001$ \\
\hline Male sex & $29(67)$ & $1(25)$ & 0.124 \\
\hline
\end{tabular}

Patients with advanced directives were not included; Abbreviations: ARDS, acute respiratory distress syndrome; COVID-19, coronavirus disease 2019; DIC, disseminated intravascular coagulation; ICU, intensive care unit; IMV, invasive mechanical ventilation.

\subsection{Weaning Off Invasive Mechanical Ventilation}

Critically ill patients exhibited a median arterial partial pressure of oxygen/fraction of inspired oxygen $\left(\mathrm{PaO}_{2} / \mathrm{FiO}_{2}\right)$ ratio of 174 , which is consistent with the oxygenation level of moderate ARDS. Precisely $38 \%$ of these patients had severe ARDS. The univariate analysis of risk factors associated with the weaning off the IMV is shown in Supplementary Table S1. IL-6 $>65 \mathrm{pg} / \mathrm{mL}$ and male sex were associated with the lowest statistically significant likelihood of weaning off IMV (Figure 2). Twenty-five percent of patients with severe ARDS weaned off IMV within eleven days versus five days for those without severe ARDS (Figure 3).

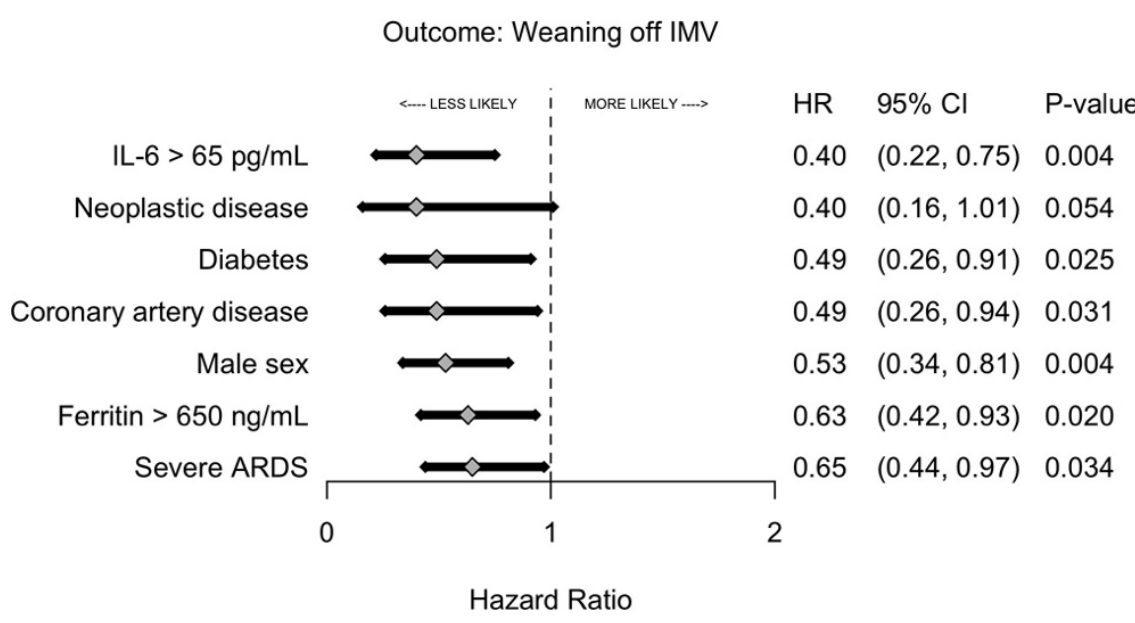

Figure 2. Likelihood of weaning off invasive mechanical ventilation by risk factors. The forest plot analysis is a multivariable logistic regression with cluster-robust standard errors that was selected using a forward and backward stepwise selection procedure based on overall model likelihood. Abbreviations: ARDS, acute respiratory distress syndrome; IL-6, interleukin-6; IMV, invasive mechanical ventilation. 


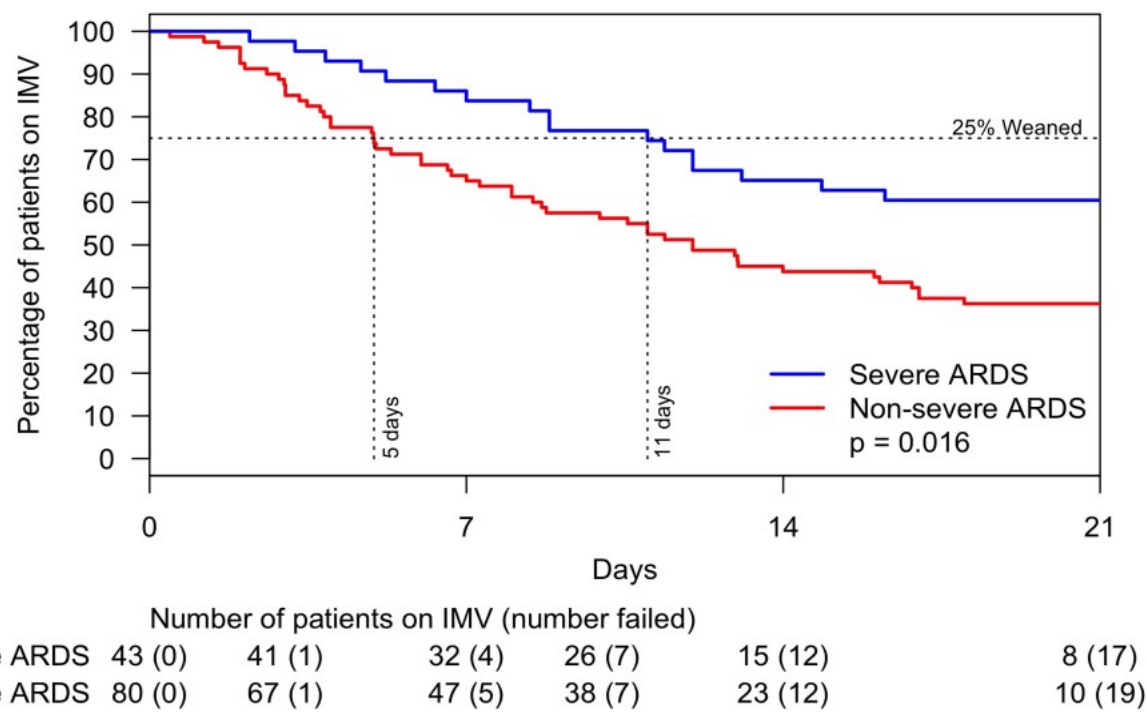

Figure 3. Likelihood of weaning off invasive mechanical ventilation within 21 days by severe and non-severe ARDS. Abbreviations: ARDS, acute respiratory distress syndrome; IMV, invasive mechanical ventilation.

\subsection{Mortality}

The overall mortality was $20 \%$ versus $1 \%$ for critically and non-critically ill patients, respectively. Among those who expired, critically ill patients were 17 years younger than those who were non-critically ill, and the male to female ratio was 2.68. The univariate analysis of risk factors associated with hospital mortality is shown in Supplementary Table S2. After stepwise selection for time to hospital mortality and adjustment by age, African American patients had a 53\% lower risk of death (Figure 4). Additionally, arterial hypertension, severe ARDS, and an increase in serum sodium were significantly associated with risk of hospital mortality. Mortality difference between patients with and without severe ARDS was minimal within the first seven days but became substantially wider thereafter. By Day $14,12 \%$ of patients without severe ARDS expired compared with $31 \%$ of patients with severe ARDS, $p=0.009$ (Figure 5).

\section{Outcome: Mortality}

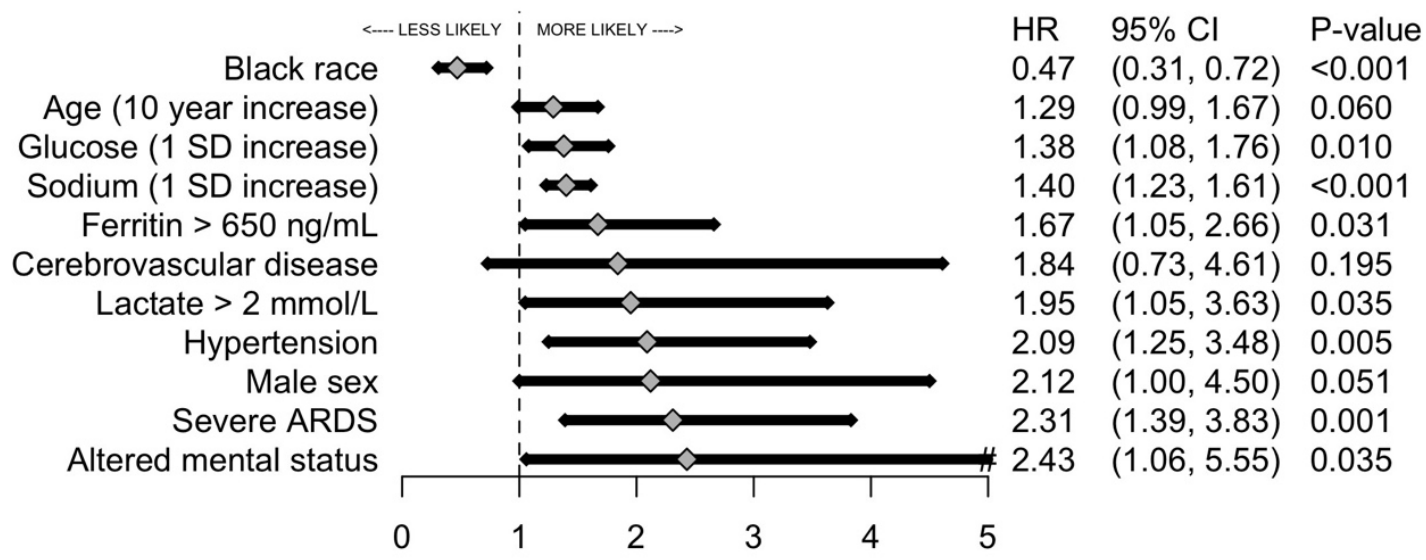

Hazard Ratio

Figure 4. Likelihood of mortality of patients with critical COVID-19 pneumonia by risk factors. The forest plot analysis is a multivariable logistic regression with cluster-robust standard errors that was selected using a forward and backward stepwise selection procedure based on overall model likelihood. Abbreviations: ARDS, acute respiratory distress syndrome; COVID-19, coronavirus disease 2019. 


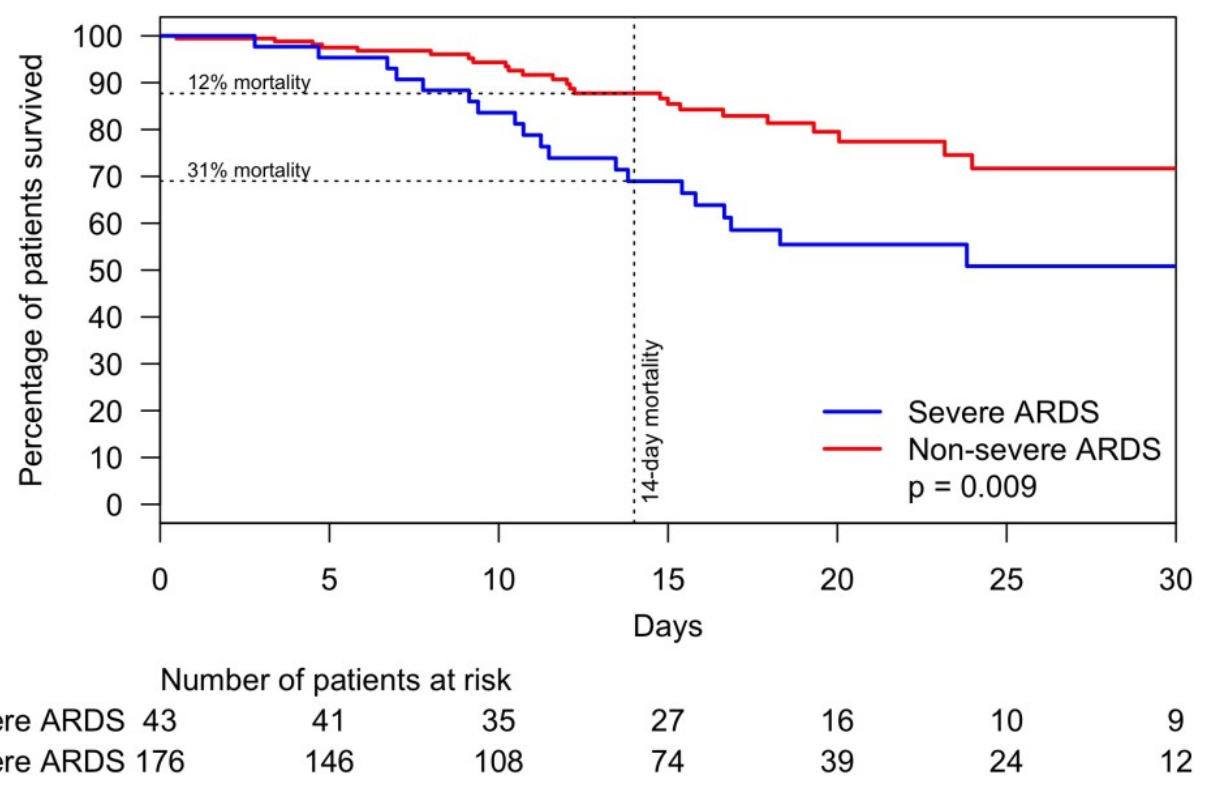

Figure 5. Time to mortality of patients with COVID-19 pneumonia within 28 days by severe and non-severe ARDS. Abbreviations: ARDS, acute respiratory distress syndrome; COVID-19, coronavirus disease 2019.

\section{Discussion}

\subsection{Patients Characteristics}

Our study demonstrated that $41.9 \%$ of patients with COVID-19 pneumonia developed critical illness, which was largely associated with ARDS. The main characteristics of our critically ill patients included a median age of 60 years, male sex (53\%), Black $(32 \%)$, White $(55 \%)$, current or former tobacco smoker $(37 \%)$, and diabetes mellitus (36\%). Therefore, a 60-year-old male who is a former or current smoker suffering from diabetes mellitus seems to be at high risk of becoming critically ill with COVID-19 pneumonia. Considering the racial distribution of the city of Louisville by the 2019 US census, African Americans were overrepresented by $10 \%$ and Whites were underrepresented by $16 \%$ among those suffering from critical COVID-19 pneumonia.

In relation to the sex distribution for critical illness, females were $7 \%$ more prevalent among those with non-critical illness and 3\% less among those with critical illness. The $10 \%$ increase in male sex from non-critical to critical COVID-19, and vice versa for female sex, demands further research. A lower percentage of females in relation to the severity of illness has been similarly reported by others [3,10-12]. This raises the binary question: are men at greater risk for critical COVID-19 pneumonia, or are women better protected against it? Female protection against critical COVID-19 pneumonia has been linked to the estrogen immunomodulatory and anti-inflammatory activity of high physiological concentrations of the steroids $17 \beta$-estradiol (E2) and progesterone (P4). It has been postulated that E2 and $\mathrm{P} 4$ decrease innate immune inflammatory responses and increase immune tolerance and antibody production. Thus, E2 and P4 may lessen the immune dysregulation that leads to the COVID-19 cytokine storm [13]. Many research studies on the effects of estrogen and COVID-19 are currently underway [14].

\subsection{ARDS and the Time to Wean Off IMV of Patients with Critical COVID-19 Pneumonia}

Our study showed that over half (52\%) of critically ill patients had ARDS, and almost one third $(28 \%)$ had severe ARDS. Furthermore, among patients who were weaned off IMV ( $25 \%$ of the study cohort), those with severe ARDS required IMV for a longer period than those with non-severe ARDS ( 15 versus 8 days, $p=0.016$ ). Since the pre-COVID era, late weaning from IMV has been associated with poor subsequent quality of life [15]. The severity of ARDS and length of IMV-dependent time exhibited by patients in our study bear some similarity to non-COVID-19 pneumonia ARDS, including influenza ARDS. The 
Lung Safe Study reported ARDS in 59.4\% of patients with pneumonia, severe ARDS in $23.43 \%$, and a median IMV-dependence period of 8 days [16]. Limited data on influenza pneumonia and ARDS are available in the literature. In a comparison of critical COVID19 pneumonia and influenza in a cohort of 201 adults, Hernu et al. reported a mean of IMV-dependence of 14 days; $56 \%$ of the patients had ARDS and $75 \%$ of those with ARDS expired [17]. Martinez et al. studied 595 patients with influenza who were admitted to the ICU; the mean age was 59 years, $37.8 \%$ were male, pneumonia was present in $34.5 \%$, and the mean period of IMV-dependence was 14 days. In this study, ARDS was present in $51.9 \%$ of patients and mortality was $20.3 \%$ among those with ARDS [18]. The higher rate of ARDS in patients with COVID-19 and the long period of IMV-dependence among our patients with severe ARDS are likely due to the higher prevalence of comorbidities associated with chronic inflammation and the not fully identified mechanisms of exuberating lung inflammation $[19,20]$. In our study, IL-6 and male sex were associated with failing to wean off IMV. IL-6 has been reported to be an independent and significant predictor of disease severity and death [21]. IL-6 was related to ACE-2 activity, which has been directly associated with the cytokine storm, and ACE-2 polymorphism could explain the variability of the rate of ARDS among patients and its lower incidence among female sex [22].

\subsection{Mortality among COVID-19 Pneumonia}

The mortality of patients in our study with critical illness was about 20 times that of patients without critical illness: $20 \%$ versus $1 \%$ (see Table 2). Furthermore, mortality was more notable and statistically significant after Day 7. The cytokine storm and, in particular, the high level of IL-6 have been strongly correlated with mortality [23]. The greater mortality of critical COVID-19 pneumonia is very likely related to the severity of illness among people suffering from comorbidities associated with chronic inflammation and ongoing organ damage [24]. Our patients with critical COVID-19 presented with more than three times as many cardiac events as those without critical COVID-19. The association of male sex, high IL-6, and ARDS could explain the higher mortality of patients with critical COVID-19.

The greatest strength of our study is its characterization of the critical illness and outcomes of patients with COVID-19 pneumonia across an entire city that is demographically representative of the US population. The large number of patients included in our study is an additional strength. The limitations of the study include the retrospective study design and missing data concerning some of the patients' characteristics.

In summary, more than two fifths (41.9\%) of patients admitted with COVID-19 pneumonia became critically ill and more than half (52\%) of them developed ARDS. Among the $25 \%$ of patients who were weaned off IMV, those with severe ARDS required about two weeks on IMV, compared to about one week for those with non-severe ARDS. The two-week mortality of patients with severe ARDS (31\%) was more than double that of patients without severe ARDS (12\%). Future research should focus on signaling mechanisms leading to deleterious inflammation and ARDS resulting in a high morbidity and mortality of patients with COVID-19 pneumonia.

Supplementary Materials: The following are available online at https: / www.mdpi.com/article / 10.3390/jcm10132935/s1, File S1: Human Subjects Protection, Data Collection and Study Coordinating Center, Table S1: Univariate Analysis of Risk Factors Associated with the Weaning off the Invasive Mechanical Ventilation, Table S2: Univariate Analysis of Risk Factors Associated with Hospital Mortality.

Author Contributions: J.B. takes responsibility for the content of the manuscript, including the data and analysis. J.B., O.A., R.S.C., S.S. and J.A.R. contributed to study concept, data analysis and interpretation, writing, and editing of the manuscript. S.F. contributed to data analysis and interpretation, writing, and editing of the manuscript. B.S. and A.A. contributed to data interpretation, writing, and editing of the manuscript. All authors have read and agreed to the published version of the manuscript. 
Funding: This research received no external funding.

Institutional Review Board Statement: The study was approved by the Institutional Review Board (IRB) at the University of Louisville Human Subjects Research Protection Program Office (IRB number 20.0257) and by the research offices at each participating hospital.

Informed Consent Statement: This study was exempt from informed consent.

Data Availability Statement: Summary data are included in the Supplementary Materials section of the online article. Other data are available on request.

Acknowledgments: Authors acknowledge the Center for Excellence for Research in Infectious Diseases (CERID) members for their discussion and input.

Conflicts of Interest: The authors declare no conflict of interest.

\section{References}

1. Johns Hopkins Coronavirus Resource Center. Available online: https:/ / coronavirus.jhu.edu (accessed on 22 June 2021).

2. Suleyman, G.; Fadel, R.A.; Malette, K.M.; Hammond, C.; Abdulla, H.; Entz, A.; Demertzis, Z.; Hanna, Z.; Failla, A.; Dagher, C.; et al. Clinical Characteristics and Morbidity Associated With Coronavirus Disease 2019 in a Series of Patients in Metropolitan Detroit. JAMA Netw. Open 2020, 3, e2012270. [CrossRef] [PubMed]

3. Petrilli, C.M.; Jones, S.A.; Yang, J.; Rajagopalan, H.; O’Donnell, L.; Chernyak, Y.; Tobin, K.A.; Cerfolio, R.J.; Francois, F.; Horwitz, L.I. Factors associated with hospital admission and critical illness among 5279 people with coronavirus disease 2019 in New York City: Prospective cohort study. BMJ 2020, 369, m1966. [CrossRef] [PubMed]

4. Furmanek, S.P.; Glick, C.; Chandler, T.; Tella, M.A.; Mattingly, W.A.; Ramirez, J.A.; Wiemken, T.L. The city of Louisville encapsulates the United States demographics. Univ. Louisville J. Respir. Infect. 2020, 4, Article 4. [CrossRef]

5. United States Census Bureau: QuickFacts. Available online: https://www.census.gov/quickfacts/fact/table/jeffersoncountykentucky\%2CUS / PST045219 (accessed on 22 June 2021).

6. Bordon, J.; Cavallazzi, R.; Furmanek, S.; Aboelnasr, A.; Tella, M.; Mattingly, W.; Wilde, A.; Sangroula, D.; Antimisiaris, D.; Chung, D.; et al. Characteristics and outcomes of adults hospitalized with SARS-CoV-2 community-acquired pneumonia in Louisville, Kentucky. Univ. Louisville J. Respir. Infect. 2020, 4, 72. [CrossRef]

7. COVID-19 Treatment Guidelines. Available online: https://www.covid19treatmentguidelines.nih.gov/overview/clinicalpresentation/ (accessed on 1 December 2020).

8. Satici, C.; Demirkol, M.A.; Sargin Altunok, E.; Gursoy, B.; Alkan, M.; Kamat, S.; Demirok, B.; Surmeli, C.D.; Calik, M.; Cavus, Z.; et al. Performance of pneumonia severity index and CURB-65 in predicting 30-day mortality in patients with COVID19. Int. J. Infect. Dis. 2020, 98, 84-89. [CrossRef]

9. Guo, J.; Zhou, B.; Zhu, M.; Yuan, Y.; Wang, Q.; Zhou, H.; Wang, X.; Lv, T.; Li, S.; Liu, P.; et al. CURB-65 may serve as a useful prognostic marker in COVID-19 patients within Wuhan, China: A retrospective cohort study. Epidemiol. Infect. 2020, 148, e241. [CrossRef] [PubMed]

10. Garibaldi, B.T.; Fiksel, J.; Muschelli, J.; Robinson, M.L.; Rouhizadeh, M.; Perin, J.; Schumock, G.; Nagy, P.; Gray, J.H.; Malapati, H.; et al. Patient Trajectories Among Persons Hospitalized for COVID-19: A Cohort Study. Ann. Intern. Med. 2021, 174, 33-41. [CrossRef] [PubMed]

11. Grasselli, G.; Zangrillo, A.; Zanella, A.; Antonelli, M.; Cabrini, L.; Castelli, A.; Cereda, D.; Coluccello, A.; Foti, G.; Fumagalli, R.; et al. Baseline Characteristics and Outcomes of 1591 Patients Infected With SARS-CoV-2 Admitted to ICUs of the Lombardy Region, Italy. JAMA 2020, 323, 1574-1581. [CrossRef]

12. Zangrillo, A.; Beretta, L.; Scandroglio, A.M.; Monti, G.; Fominskiy, E.; Colombo, S.; Morselli, F.; Belletti, A.; Silvani, P.; Crivellari, M.; et al. Characteristics, treatment, outcomes and cause of death of invasively ventilated patients with COVID19 ARDS in Milan, Italy. Crit. Care. Resusc. 2020, 22, 200-211. [PubMed]

13. Lazartigues, E.; Qadir, M.M.F.; Mauvais-Jarvis, F. Endocrine Significance of SARS-CoV-2's Reliance on ACE2. Endocrinology 2020, 161. [CrossRef]

14. Maleki Dana, P.; Sadoughi, F.; Hallajzadeh, J.; Asemi, Z.; Mansournia, M.A.; Yousefi, B.; Momen-Heravi, M. An Insight into the Sex Differences in COVID-19 Patients: What are the Possible Causes? Prehosp. Disaster. Med. 2020, 35, 438-441. [CrossRef] [PubMed]

15. Depuydt, P.; Oeyen, S.; De Smet, S.; De Raedt, S.; Benoit, D.; Decruyenaere, J.; Derom, E. Long-term outcome and health-related quality of life in difficult-to-wean patients with and without ventilator dependency at ICU discharge: A retrospective cohort study. BMC. Pulm. Med. 2016, 16, 133. [CrossRef]

16. Bellani, G.; Laffey, J.G.; Pham, T.; Fan, E.; Brochard, L.; Esteban, A.; Gattinoni, L.; van Haren, F.; Larsson, A.; McAuley, D.F.; et al. Epidemiology, Patterns of Care, and Mortality for Patients With Acute Respiratory Distress Syndrome in Intensive Care Units in 50 Countries. JAMA 2016, 315, 788-800. [CrossRef] 
17. Hernu, R.; Chroboczek, T.; Madelaine, T.; Casalegno, J.S.; Lina, B.; Cour, M.; Argaud, L. Early oseltamivir therapy improves the outcome in critically ill patients with influenza: A propensity analysis. Intensive Care. Med. 2018, 44, 257-260. [CrossRef] [PubMed]

18. Martínez, A.; Soldevila, N.; Romero-Tamarit, A.; Torner, N.; Godoy, P.; Rius, C.; Jané, M.; Domínguez, À. Risk factors associated with severe outcomes in adult hospitalized patients according to influenza type and subtype. PLoS ONE 2019, 14, e0210353. [CrossRef] [PubMed]

19. Du, R.H.; Liang, L.R.; Yang, C.Q.; Wang, W.; Cao, T.Z.; Li, M.; Guo, G.Y.; Du, J.; Zheng, C.L.; Zhu, Q.; et al. Predictors of mortality for patients with COVID-19 pneumonia caused by SARS-CoV-2: A prospective cohort study. Eur. Respir. J. 2020, 55. [CrossRef]

20. Gupta, S.; Hayek, S.S.; Wang, W.; Chan, L.; Mathews, K.S.; Melamed, M.L.; Brenner, S.K.; Leonberg-Yoo, A.; Schenck, E.J.; Radbel, J.; et al. Factors Associated With Death in Critically Ill Patients With Coronavirus Disease 2019 in the US. JAMA Intern. Med. 2020, 180, 1436-1447. [CrossRef] [PubMed]

21. Del Valle, D.M.; Kim-Schulze, S.; Huang, H.H.; Beckmann, N.D.; Nirenberg, S.; Wang, B.; Lavin, Y.; Swartz, T.H.; Madduri, D.; Stock, A.; et al. An inflammatory cytokine signature predicts COVID-19 severity and survival. Nat. Med. 2020, 26, 1636-1643. [CrossRef]

22. Thompson, B.T.; Chambers, R.C.; Liu, K.D. Acute Respiratory Distress Syndrome. N. Engl. J. Med. 2017, 377, 562-572. [CrossRef]

23. Fajgenbaum, D.C.; June, C.H. Cytokine Storm. N. Engl. J. Med. 2020, 383, 2255-2273. [CrossRef]

24. Wu, C.; Chen, X.; Cai, Y.; Xia, J.; Zhou, X.; Xu, S.; Huang, H.; Zhang, L.; Zhou, X.; Du, C.; et al. Risk Factors Associated With Acute Respiratory Distress Syndrome and Death in Patients With Coronavirus Disease 2019 Pneumonia in Wuhan, China. JAMA Intern. Med. 2020, 180, 934-943. [CrossRef] [PubMed] 